\title{
Temperature and Molecular Weight Effect of Styrene-Isoprene Diblock Copolymers on the Interfacial Tension between Polystyrene and Polyisoprene
}

\author{
Kie Hyun Nam, Jae Cheol Cho, and Won Ho Jo* \\ Department of Fiber and Polymer Science, Seoul National University, \\ Seoul 151-742, Korea
}

(Received January 20, 1995)

\begin{abstract}
The effect of styrene-isoprene diblock copolymers on the interfacial tension between polystyrene (PS) and polyisoprene (PI) has been investigated by direct measurement of the interfacial tension using a pendant drop interfacial tension apparatus. The interfacial tension is nearly independent of the temperature when the diblock copolymers are added, although the interfacial tension of PS/PI without copolymers decreases with increasing the temperature. A decrease in interfacial tension is observed with the addition of a small amount of copolymer, followed by a leveling off when the copolymer concentration exceeds a critical micelle concentration. The critical micelle concentration increases with temperature. The effect of the molecular weight of block copolymers on the interfacial tension is not significant.

KEY WORDS Diblock Copolymer / Interfacial Tension / Pendant Drop

Method / Critical Micelle Concentration / Molecular Weight Effect /
\end{abstract}

In heterogeneous polymer blend systems, physical or chemical interactions across the phase boundaries, which determine the interfacial tension, are known to control phase morphology. Strong interaction results in good adhesion and makes the stress transfer from the continuous to the dispersed phase in the blends effective. Unfortunately, that is not the case for most immiscible polymer blends. To overcome this weak point, it is required to use a third component (compatibilizer) capable of specific interactions or chemical reactions with the blend components. ${ }^{1}$ Nowaday it is widely known that the addition of a proper graft or block copolymer can alleviate to some extent week points of immiscible polymer blends. ${ }^{1-7}$ The interfacial activity of block copolymers has been the most attractive subject in this field because the molecular weight, the polydispersity and the structure of the block co-

\footnotetext{
* To whom correspondence should be addressed.
}

polymer can be easily controlled by anionic polymerization methods. The interfacial activity of a block copolymer in homopolymer blends is assessed most clearly by the reduction in the interfacial tension between the two immiscible phases. Interfacial tension is the single most accessible parameter that describes the thermodynamic state and structure of an interface. Moreover, interfacial tension is very important because it has much to do with the phase morphology, the interfacial adhesion, thus affecting the mechanical properties of the immiscible polymer blends. ${ }^{8}$

Several theoretical and experimental studies on the segregation of diblock copolymers to the interface between two homopolymers have been reported. It has been found experimentally that the interfacial tension decreases with increasing the concentration of block copolymer and that block copolymer micelles are 
formed when the copolymer concentration in the homopolymer phase exceeds the critical micelle concentration (CMC). Patterson et al. ${ }^{9}$ used a rotating drop apparatus to study the effect of addition of poly(dimethyl siloxane- $b$ oxyethylene) on the interfacial tension between poly(dimethyl siloxane) and poly(oxyethylene$b$-oxypropylene). They directly measured the interfacial tension reduction caused by the addition of the copolymer as an interfacial agent, and found that the interfacial tension reduces from 8.3 to $2.5 \mathrm{dyn} \mathrm{cm}^{-1}$ with the addition of $2 \mathrm{wt} \%$ of a poly(dimethyl siloxane$b$-oxyethylene). The CMC was found at $c a$. $1 \mathrm{wt} \%$ and further addition of the block copolymer are not effective in reducing the interfacial tension. However, no information about the polymer characteristics such as the molecular weight and its polydispersity of the block copolymers and blend components was not reported.

The compatibilizing effect of poly(styrene- $b$ 1,2-butadiene) on the interfacial tension between polystyrene and 1,2-polybutadiene was studied as a function of the concentration of the block copolymer by using the pendant drop method. ${ }^{10} \mathrm{~A}$ significant decrease in interfacial tension was observed with the addition of a low concentration of copolymer $(<1.5 \mathrm{wt} \%)$.

In this study, the effect of styrene-isoprene diblock copolymers on the interfacial tension between polystyrene and polyisoprene has been investigated as a function of temperature and molecular weight of block copolymers by direct measurement of the interfacial tension using a pendant drop interfacial tension apparatus.

\section{EXPERIMENTAL}

\section{Materials}

The polyisoprene (PI) $\left(\bar{M}_{n} \cong 9800 \mathrm{~g} \mathrm{~mol}^{-1}\right)$ and polystyrene (PS) $\left(\bar{M}_{n} \cong 5100 \mathrm{~g} \mathrm{~mol}^{-1}\right)$ were anionically synthesized in cyclohexane at room temperature using $s$-butyllithium $(s-\mathrm{BuLi})$ as an initiator. Three different molecular weight styrene-isoprene diblock copolymers (SI4, SI10, $\operatorname{SI} 16)\left(\bar{M}_{n} \cong 41000,95000,162000 \mathrm{~g} \mathrm{~mol}^{-1}\right)$ were synthesized by sequential anionic polymerization of styrene and isoprene in benzene using $s$-BuLi as an initiator. All the SI block copolymers are nearly symmetric and monodisperse. The number average molecular weight and the polydispersity of polymers were measured by gel permeation chromatography (see Table I).

\section{Interfacial Tension Measurement}

Interfacial tensions were measured by using a pendant drop apparatus made in our laboratory. The apparatus consists of a heated sample holder in which a pendant drop is formed, an optical system to capture the image of the drop, and a data acquisition system with a Macintosh computer. The optical system consists of the light source (halogen lamp and optical fiber) and the CCD video camera. A zooming lens is coupled to the CCD camera. The sample holder for the pendant drop consists of an electrically heated cylindrical block with the chamber in which the quartz cell can be placed. The syringe is inserted into a fluid matrix of PI which is contained in a quartz microcuvette. A fluid drop of the more

Table I. Polymer characteristics

\begin{tabular}{lccc}
\hline \multicolumn{1}{c}{ Polymers } & Abbreviation & $M_{n} / \mathrm{g} \mathrm{mol}^{-1 \mathrm{a}}$ & Polydispersity $^{\mathrm{b}}$ \\
\hline Polystyrene & PS & 5100 & 1.06 \\
Polyisoprene & PI & 9800 & 1.13 \\
Styrene-isoprene & SI4 & 41000 & 1.19 \\
Block copolymer & SI10 & 95000 & 1.14 \\
& SI16 & 162000 & 1.02 \\
\hline
\end{tabular}

${ }^{\mathrm{a}, \mathrm{b}}$ Determined from GPC. 
dense constituent (PS) is formed at the tip of a glass capillary tube of a syringe made of Pyrex. The block copolymer was preblended with the dispersed phase, PS, in order to control the $\mathrm{wt} \%$ of a copolymer precisely and to minimize the distance traveled by the copolymer chains to reach the polymer/polymer interface. The blends were prepared by dissolving the copolymer and PS in tetrahydrofuran (THF), precipitating into methanol and drying. The argon gas is purged into the chamber containing the quartz microcuvette to avoid oxidation and degradation of polyisoprene. The temperature of the chamber is controlled within $\pm 1^{\circ} \mathrm{C}$. An image of the drop is obtained and digitized by a frame grabber in a Macintosh computer. Interfacial tension is calculated from the equation developed by Andreas et al. ${ }^{11}$ The equation contains the densities of polystyrene and polyisoprene, which are taken from the data of Fox and Flory's paper, ${ }^{12}$ and the data in "Polymer Handbook,"13 respectively. All the data are reported by averaging the results of three tests. The experimental deviation lies between 2 and $7 \%$ of the reported values. When our data for PS/PI from the Andreas' method are compared with the data for PS/HPB (hydrogenated polybutadiene) from the other method, ${ }^{14}$ both methods give almost the same magnitude of interfacial tension and its temperature dependence. Since the interaction parameter $\chi$ between PS and PI is almost the same as that between PS and HPB, we believe that the Andreas' method could give reliable data.

\section{RESULTS AND DISCUSSION}

\section{Temperature Dependence of the Interfacial Tension}

The temperature dependence of interfacial tension between PS and PI are shown in Figure 1. The interfacial tension between PS and PI without a block copolymer decreases with increasing temperature, whereas the interfacial tension of the system containing the SI 4 block

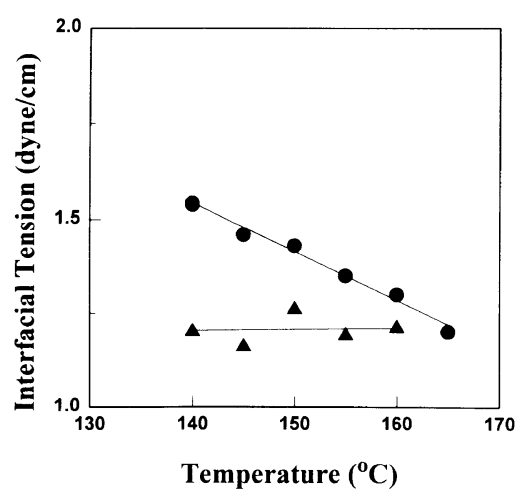

Figure 1. Interfacial tension as a function of temperature for the system PS/SI4/PI: (O) without SI; (A) $0.5 \mathrm{wt} \%$ of SI4 added into PS.

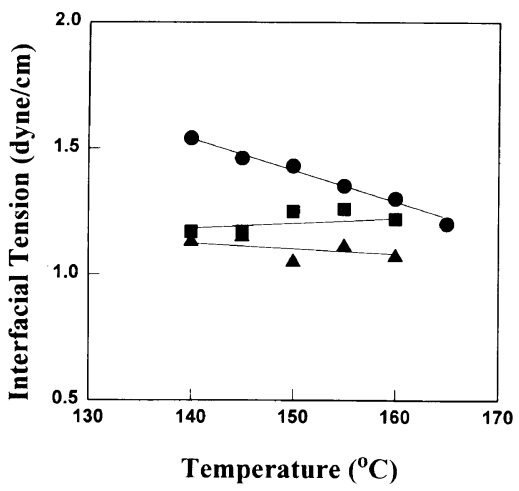

Figure 2. Interfacial tension as a function of temperature for the system PS/SI10/PI: (O) without SI 10; (ם) $0.5 \mathrm{wt} \%$ of SI 10 added into PS; (A) $1 \mathrm{wt} \%$ of SI 10 added into PS.

copolymer does not change significantly with the temperature. Furthermore, the difference between the interfacial tensions of systems with and without the block copolymer is significant at relatively lower temperature $\left(<150^{\circ} \mathrm{C}\right)$, but the difference decreases to near zero with increasing temperature. In other words, the interfacial tension between PS and PI is significantly reduced by the addition of a small amount of block copolymer at relatively lower temperatures, but the effect of the block copolymer on the interfacial tension becomes less significant as the temperature increases. Similar tendencies are also observed when the SI10 is added to PS/PI system, as shown in Figure 2. These phenomena may be explained 
by the following consideration and theoretical analysis.

In an immiscible $\mathrm{A} / \mathrm{B}$ polymer system containing a small amount of $\mathrm{A}-\mathrm{B}$ block copolymer as an interfacial agent, the reduction of interfacial tension arises mainly from the fact that the segregation of the copolymer to the interface reduces the interaction energy between $\mathrm{A}$ and $\mathrm{B}$ homopolymer. Therefore, it is generally accepted that the extent to which the interfacial tension is reduced is proportional to the Flory-Huggins interaction parameter $(\chi)$. The $\chi$ parameter is very often expressed as eq 1, in order to explain the temperature dependence of the parameter, i.e., the $\chi$ decreases with increasing temperature in an immiscible polymer blend:

$$
\chi=A+\frac{B}{T}
$$

where $A$ and $B$ are constants that depend on the pair of the polymer blend. As a result, the interfacial tension reduction $(\Delta \gamma)$ is reduced as the temperature increases. In the limit case, i.e., when the temperature reaches the critical temperature, the effect of the copolymer as an interfacial agent vanishes.

Noolandi and Hong ${ }^{15,16}$ proposed statistical thermodynamic theories for the emulsifying effect of block copolymers. They derived the mean-field equations for the fundamental probability distribution functions for a system of two immiscible homopolymers, $A$ and $B$, diluted with solvent in the presence of a $A-B$ diblock copolymer. These equations were solved numerically to obtain the polymer density profiles in the interfacial region. The difference between the total free energy of the blend and that of the bulk polymers was used to evaluate the interfacial tension. It was assumed that the part of the copolymer of not localizing itself at the interface will be randomly distributed in the bulk of the homopolymers. Because of this assumption, their results should be reliable only for low copolymer concentrations below the critical micelle concentration.
Neglecting the loss of conformational entropy, they were able to obtain an analytical expression for the interfacial tension reduction $(\Delta \gamma)$, given by

$$
\begin{aligned}
\Delta \gamma=\gamma-\gamma_{0}= & \mathrm{d} \phi_{\mathrm{c}}\left\{\left(\frac{1}{2} \chi \phi_{\mathrm{p}}+\frac{1}{Z_{\mathrm{c}}}\right)\right. \\
& \left.-\frac{1}{Z_{\mathrm{c}}} \exp \left(\frac{Z_{\mathrm{c}} \chi \phi_{\mathrm{p}}}{2}\right)\right\}
\end{aligned}
$$

where $\gamma_{0}$ is the interfacial tension of the blend system without a block copolymer, and $d$ is the width at half height of the copolymer profile, reduced by the Kuhn statistical segment length. Numerical calculations showed that $d$ was almost constant for the molecular weight of the copolymer. In eq $1, \phi_{\mathrm{c}}$ denotes the bulk volume fraction of the copolymer in the system, $\phi_{\mathrm{p}}$ is the bulk volume fraction of polymer $A$ and $B$ (assumed equal), $Z_{\mathrm{c}}$ is the degree of polymerization of the symmetric copolymer, and $\chi$ is the Flory-Huggins interaction parameter between $A$ and $B$ segments. It was assumed that the interaction parameters between segments and solvent, $\chi_{\mathbf{A S}}=\chi_{\mathbf{B S}}=0$. It was suggested that the theory can be applied to a concentrated system without solvent by letting the total polymer volume fraction $\left(\phi_{\mathrm{p}}\right)$ go to unity. ${ }^{10}$ In this case, eq 2 reduces to

$$
\Delta \gamma=\mathrm{d} \phi_{\mathrm{c}}\left\{\frac{1}{2} \chi+\frac{1}{Z_{\mathrm{c}}}-\frac{1}{Z_{\mathrm{c}}} \exp \left(\frac{Z_{\mathrm{c}} \chi}{2}\right)\right\}
$$

From eq 3 , it is seen that the interfacial tension reduction $(\Delta \gamma)$ strongly depends on the amount of block copolymers added and the FloryHuggins interaction parameter $\chi$. In eq 3 , we realize that the only parameter depending on temperature is $\chi$. Thus, the interfacial tension reduction as a function of temperature can be predicted when eq 1 is substituted into eq 3 .

In order to apply the theory to our system, the bulk volume fraction of the copolymer and the number of Kuhn's statistical segments $\left(Z_{\mathrm{cs}}\right)$ should be known. The number $Z_{\mathrm{cs}}$ is estimated from the characteristic ratios tabulated in a literature. ${ }^{13}$ It is also assumed that the blend 


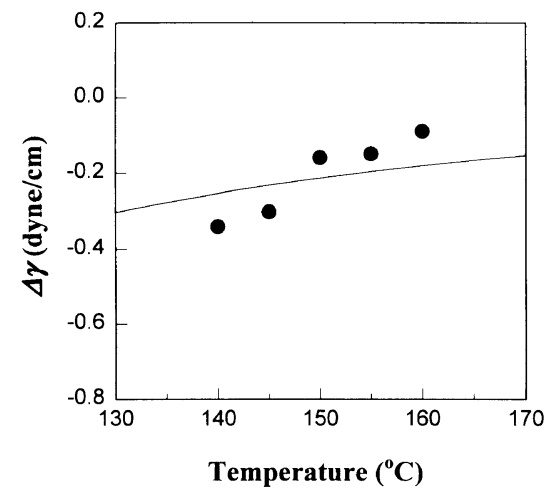

Figure 3. Comparison of theoretical prediction with experimental results for the temperature dependence of interfacial tension reduction $(\Delta \gamma)$ when $0.5 \mathrm{wt} \%$ of SI 4 is added. The solid line is drawn from eq 3 , and the filled circles represent the experimental data.

ratio of two homopolymers is 5/5 and the same volume fraction of copolymer exists in both bulk phases. When the experimentally determined values of $A$ and $B$ for PS/PI systems, $A=-0.0937$ and $B=66,{ }^{17}$ are used, the temperature dependence of the interfacial tension $(\Delta \gamma / \Delta T)$ can be predicted. The interfacial tension reduction $(\Delta \gamma)$ as a function of temperature are shown in Figure 3, when $0.5 \mathrm{wt} \%$ of SI 4 is added. The theoretical prediction is well consistent with experimental data. The reported values of $A$ and $B$ for the styrene-isoprene diblock copolymer synthesized in THF may be different from the values of the copolymer used in this paper since the block copolymer used in this paper were synthesized in cyclohexane and the polyisoprene block has 1,4-type microstructure. However, the difference is probably not large enough to change the results.

\section{Critical Micelle Concentration}

It is generally accepted that the interfacial tension of the immiscible polymer blend decreases linearly with the concentration of block copolymers added and then levels off when the concentration exceeds the CMC. The CMC strongly depends on the temperature, the structure (the molecular weight, the block ratio

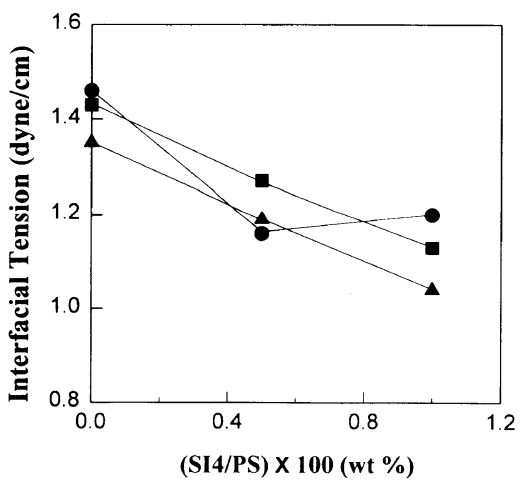

Figure 4. The effect of copolymer concentration on the interfacial tension for the system PS/SI4/PI: (O) $145^{\circ} \mathrm{C}$; (a) $150^{\circ} \mathrm{C} ;(\boldsymbol{\Delta}) 155^{\circ} \mathrm{C}$.

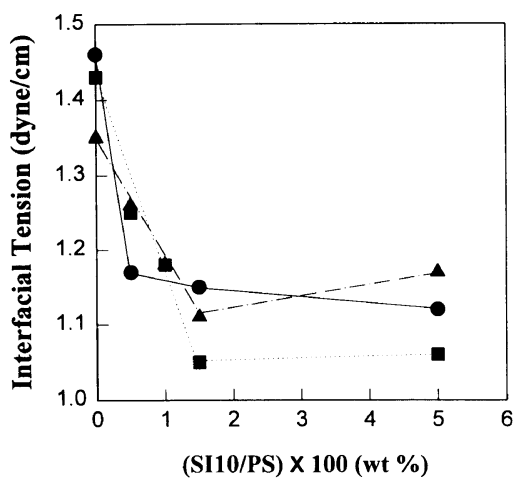

Figure 5. The effect of copolymer concentration on the interfacial tension for the system PS/SI10/PI: (O) $145^{\circ} \mathrm{C}$; (更) $150^{\circ} \mathrm{C} ;(\mathbf{\Delta}) 155^{\circ} \mathrm{C}$.

and the molecular architecture) of the block copolymer added and the molecular weight of homopolymers. Figure 4 shows the dependence of the interfacial tension on the copolymer concentration at various temperature for PS/SI4/PI system. The interfacial tension decreases with increasing the copolymer concentration up to $0.5 \mathrm{wt} \%$ at $145^{\circ} \mathrm{C}$, while it tends to decrease continuously till $1 \mathrm{wt} \%$ at 150 and $155^{\circ} \mathrm{C}$. The change in the $\mathrm{CMC}$ with the temperature is shown more clearly for PS/SI10/PI system, as shown in Figure 5. The $\mathrm{CMC}$ of SI 10 at $145^{\circ} \mathrm{C}$ is about $0.5 \mathrm{wt} \%$ while it lies in the range between 1 and $1.5 \mathrm{wt} \%$ at temperatures higher than $150^{\circ} \mathrm{C}$. In other 
words, the CMC increases with the temperature. Therefore, it is envisaged that copolymer chains tend to remain freely in the bulk phase than to form micelles as the temperature increases. Roe ${ }^{18}$ observed from SAXS data that the CMC increases with the temperature for polystyrene/styrene-butadiene diblock copolymer system. Whitmore and Noolandi ${ }^{19}$ also predicted from the theory of micelle formation in block copolymer-homopolymer blends that the number of copolymer chains remaining freely in bulk phase increases with the temperature. The slope of interfacial tension versus copolymer concentration plot becomes larger in the lower concentration range than $\mathrm{CMC}$ as the temperature decreases. This phenomenon indicates that the block copolymers more easily aggregate at the interface at higher temperature as compared with the case of lower temperatures.

\section{The Molecular Weight Effect}

The effect of number average molecular weight $\left(M_{n}\right)$ of the block copolymer on the interfacial tension is given in Figure 6. The change in the interfacial tension with the molecular weight of the copolymer is not significant when $0.5 \mathrm{wt} \%$ of the block copolymer is added. This fact is not in accord with the theory of Noolandi and Hong, ${ }^{15,16}$ which explains that the interfacial tension reduction increases with the degree of polymerization of a block copolymer (eq 2). This can be explained as follows. First, the interfacial tension reduction for all the blend systems containing SI block copolymer is not large enough to distinguish the variation in the interfacial tension with the copolymer molecular weight, i.e., $-0.4 \mathrm{dyn} \mathrm{cm}^{-1}<\Delta \gamma<-0.2 \mathrm{dyn} \mathrm{cm}^{-1}$ in this system. Second, both the tendency of the block copolymer to form micelles in bulk phase and the potential of the copolymer to go to the interface become larger as the molecular weight of the copolymer increases. That is, the force of copolymer chains to aggregate at the interface is counterbalanced by the tendency

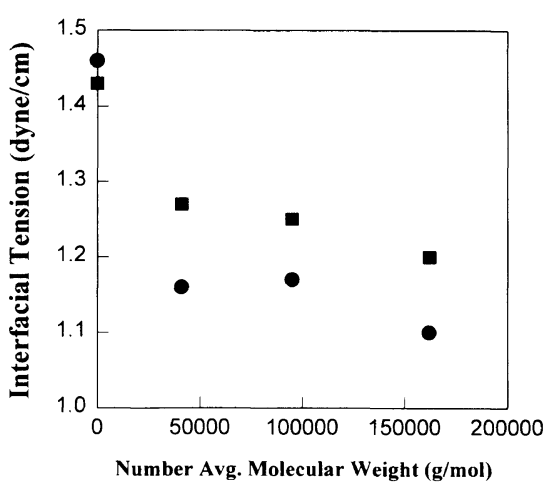

Figure 6. Interfacial tension as a function of number average molecular weight of SI for the systems PS/SI/PI

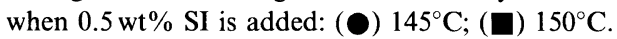

to form micelles in bulk phases of homopolymer. Noolandi and Hong ${ }^{20}$ also pointed out that the interfacial tension reduction is bounded by the CMC, since blocks of large molecular weight tend to form micelles in the bulk of the homopolymers rather than to congregate at the interface although both copolymer concentration and molecular weight are equally important in reducing the interfacial tension. Thus their theoretical treatment is valid for concentrations inside the CMC boundary.

\section{CONCLUSIONS}

The temperature and molecular weight effect of styrene-isoprene diblock copolymers on the interfacial tension between polystyrene and polyisoprene was examined by using a pendant drop interfacial tension apparatus. The interfacial tension is nearly independent of the temperature when the diblock copolymers are added, although the interfacial tension of PS/PI without copolymers decreases with increasing the temperature. As a result, the interfacial tension reduction becomes near zero at higher temperatures while a significant decrease in interfacial tension is observed with the addition of a small amount of copolymer at the temperatures lower than $150^{\circ} \mathrm{C}$. The temperature dependence of a interfacial tension of the 
blend containing the block copolymer could be well explained by the theory of Noolandi and Hong. ${ }^{20}$ The interfacial tension is reduced significantly with addition of a small amount of copolymer, followed by a leveling off as the copolymer concentration increases. It is also observed that the CMC increases with the temperature. The molecular weight effect of block copolymers on the interfacial tension is not significant. This is because both the tendency of the block copolymer to form micelles in bulk phase and the potential of the copolymer to go to the interface become larger as the molecular weight of the copolymer increases, resulting in the counterbalance of these two effects.

Acknowledgment. The authors thank the Ministry of Education, the Republic of Korea for their financial support through the Advanced Materials Program (1993).

\section{REFERENCES}

1. D. R. Paul and S. Newman, Ed., "Polymer Blends," Academic Press, New York, N.Y., 1978.

2. D. Heikens, N. Hoen, W. M. Barentsen, P. Piett, and H. Landen, J. Polym. Sci., Polym. Symp., 62, 309 (1978).
3. R. Fayt, R. Jérôme, and Ph. Teyssié, J. Polym. Sci., Polym. Phys. Ed., 20, 2209 (1982).

4. R. Fayt, R. Jérôme, and Ph. Teyssié, Makromol. Chem., 187, 837 (1986).

5. T. Ouhadi, R. Fayt, R. Jérôme, and Ph. Teyssié, $J$. Polym. Sci., Polym. Phys. Ed., 24, 1973 (1986).

6. W. H. Jo, H. C. Kim, and D. H. Baik, Macromolecules, 24, 2231 (1991).

7. H. C. Kim, K. H. Nam, and W. H. Jo, Polymer, 34, 4043 (1993).

8. S. Wu, "Polymer Interface," Marcel Dekker, New York, N.Y., 1982.

9. H. T. Patterson, K. H. Hu, and T. H. Grinstaff, $J$. Polym. Sci., C, 34, 31 (1971).

10. S. H. Anastasiadis, I. Gancarz, and J. T. Koberstein, Macromolecules, 22, 1449 (1989).

11. J. M. Andreas, E. A. Hauser, and W. B. Tucker, J. Phys. Chem., 42, 1001 (1938).

12. T. G. Fox, Jr. and P. J. Flory, J. Appl. Phys., 21, 581 (1950).

13. J. Brandup and E. H. Immergut, Ed., "Polymer Handbook," 3rd ed, Wiley, New York, N.Y., 1988.

14. S. H. Anastasiadis, I. Gancarz, and J. T. Koberstein, Macromolecules, 21, 2980 (1988).

15. J. Noolandi and K. M. Hong, Macromolecules, 15, 482 (1982).

16. J. Noolandi and K. M. Hong, Macromolecules, 17, 1531 (1984).

17. K. Mori, H. Hasegawa, and T. Hashimoto, Polym. J., 17, 799 (1985).

18. R. J. Roe, Macromolecules, 19, 728 (1986).

19. M. D. Whitmore and J. Noolandi, Macromolecules, 18, 657 (1985).

20. J. Noolandi, Polym. Eng. Sci., 24, 70 (1984). 